\title{
Dynamics of sheared droplets filled with non-Brownian particles
}

\author{
Helene Van Ammel ${ }^{1}$ - Paula Moldenaers ${ }^{1}$ - Ruth Cardinaels ${ }^{1,2}$ (D) \\ Received: 22 March 2020 / Revised: 13 August 2020 / Accepted: 7 September 2020 / Published online: 30 October 2020 \\ (C) The Author(s) 2020
}

\begin{abstract}
The dynamics of single droplets containing non-Brownian particles are studied. The particle over droplet size ratio $(r / R)$ is changed by using different particle sizes $(r / R=0.02-0.4)$. Additionally, the effect of particle concentration $(5-20$ vol\%) is investigated. The dynamics of droplets with $r / R=0.02$ show good agreement with the corresponding particle-free reference system which has a comparable viscosity ratio. Hence, this droplet phase can be considered as a homogenous medium characterized by its bulk viscosity which is governed by the particle concentration. However, droplets with $r / R \geq 0.1$ show a more suppressed deformation and slower transient dynamics and, therefore, behave as a slightly more viscous medium than expected based on their bulk viscosity. These effects become more pronounced at higher particle concentrations and higher $r / R$. Moreover, local particle effects like asymmetric droplet shapes, oscillating droplet shapes, and tip streaming start to influence the droplet dynamics at particle concentrations around $15 \mathrm{vol} \%$.
\end{abstract}

Keywords Emulsions $\cdot$ Polymer blends $\cdot$ Particle-filled systems $\cdot$ Non-Brownian particles $\cdot$ Droplet dynamics

\section{Introduction}

Polymer blends are used in many everyday products and materials to achieve superior properties as compared with their single polymer counterparts. The final properties of blends of immiscible polymers can be optimized by tailoring the phase morphology (for a recent review, see Cardinaels and Moldenaers (2016)). Over a wide composition range, such blends exhibit a droplet-matrix morphology. Therefore, the relationships between the applied flow and the single droplet dynamics of systems consisting of two Newtonian fluids have been studied extensively (Taylor 1934; Torza et al. 1972; Rallison 1984; Kennedy et al. 1994; Janssen and Anderson 2007). In this case, the droplet dynamics depend only on two dimensionless numbers: the capillary number $\mathrm{Ca}$ and the

Electronic supplementary material The online version of this article (https://doi.org/10.1007/s00397-020-01240-6) contains supplementary material, which is available to authorized users.

Ruth Cardinaels

Ruth.Cardinaels@kuleuven.be

1 Soft Matter, Rheology and Technology, Department of Chemical Engineering, KU Leuven, Box 2424, Celestijnenlaan 200F, BE 3001 Leuven, Belgium

2 Polymer Technology, Department of Mechanical Engineering, TU Eindhoven, Box 513, 5600 MB Eindhoven, The Netherlands viscosity ratio $\lambda$. The capillary number represents the balance of the deforming viscous forces induced by the applied flow and the restoring forces of the interfacial tension.

In the specific case of simple shear flow, $\mathrm{Ca}$ is given by $\mathrm{Ca}=\left(\eta_{\mathrm{m}} R \dot{\gamma}\right) / \Gamma$, with $R$ as the droplet radius, $\eta_{\mathrm{m}}$ as the matrix viscosity, $\dot{\gamma}$ as the applied shear rate, and $\Gamma$ as the interfacial tension. The viscosity ratio is the ratio of the droplet phase viscosity $\eta_{\mathrm{d}}$ over the matrix phase viscosity $\eta_{\mathrm{m}}: \lambda=\eta_{\mathrm{d}} / \eta_{\mathrm{m}}$. Maffettone and Minale (1998) developed a phenomenological model which is able to describe the droplet dynamics of ellipsoidal Newtonian droplets in a Newtonian matrix in various flow fields and shows good agreement between model predictions and experimental data (Maffettone and Minale 1998; Vananroye et al. 2007; Vananroye et al. 2008).

Solid particles are often incorporated in polymer blends to enhance the overall material performance, such as mechanical properties (Liu and Kontopoulou 2006; Wahit et al. 2006; Kelnar et al. 2007; Yang et al. 2007) or thermal stability (Hong et al. 2006b; Elias et al. 2007; Filippone et al. 2010). Furthermore, particles can introduce additional functionalities, such as electrical conductivity (Geuskens et al. 1987; Huang 2002; Bharati et al. 2015). Particles are also often added to control the blend morphology as they can act as compatibilizers when they are trapped at the interface (Ray et al. 2004; Zhang et al. 2004; Vandebril et al. 2010; Yoshida and Trifkovic 2019). Prior work on single droplets 
has already shown that particles trapped at the interface of a sheared droplet significantly suppress droplet deformation and breakup and cause hysteresis in the droplet dynamics (Mei et al. 2016).

In the present work, we focus on the effects of particles localized in the droplet phase. Several authors (Gahleitner et al. 2006; Hong et al. 2006b; Zou et al. 2006; Kontopoulou et al. 2007; Amoabeng et al. 2017) agree on the fact that the addition of particles to the dispersed phase of a polymer blend results in an increased droplet size and coarser microstructure. According to Hong et al. (2006b) and Kontopoulou et al. (2007), droplet breakup is hindered by the addition of particles to the dispersed phase. In addition, cocontinuous structures are more likely to develop when particles are present in the dispersed phase (Zou et al. 2006; Filippone et al. 2010; Nuzzo et al. 2014). In contrast with these results, Elias et al. $(2007,2008)$ report a reduced droplet size regardless of the location of the nanoparticles. They attribute this reduction to the decrease in effective interfacial tension caused by the addition of nanoparticles. However, Elias et al. $(2007,2008)$ also observed a small amount of nanoparticles trapped at the interface. To our knowledge, these nanoparticles, present at the droplet interface, can be held responsible for the reduction in interfacial tension and droplet size (Hong et al. 2006a). Filippone et al. (2010) also observed a droplet size reduction, yet only at low particle concentrations. They associated this reduction with the increased viscosity of the dispersed phase which hinders coalescence and coarsening.

Only a few studies elucidate the effects of nanoparticles localized in the dispersed phase of a polymer blend on the droplet dynamics. The addition of carbon nanotubes to the dispersed phase of a PP/PA6 blend suppresses the droplet deformation and breakup. Additionally, the dynamics to reach steady-state deformation slow down, while droplet retraction accelerates (Gooneie et al. 2015; Gooneie et al. 2016). In contrast, Kong et al. (2011) observed a retarded retraction and suppressed breakup by the addition of silica nanoparticles to the dispersed phase of a PS/PA6 blend. In both cases, the observed effects are attributed to the increased viscous and elastic properties of the dispersed phase. Numerical simulations by Usta et al. (2009) also predict a reduced deformation with increasing particle concentration due to the additional viscous stresses introduced by the suspended particles.

Besides in polymer blends, droplets encapsulating particles are also of great importance in the oil, printing, and food industry (Smith and van de Ven 1985b; Desse et al. 2009; Usta et al. 2009; Bonnoit et al. 2012; Mehrabian et al. 2015; Merkel et al. 2015). Consequently, several studies focus on the droplet dynamics of water/oil systems containing particles suspended in the dispersed phase. In an early study, Powell and Mason (1982) investigated the effect of high particle concentrations ( $\sim 50$ vol\%) on the deformation and breakup behavior. Smith and van de Ven (1985b) later extended this study to lower particle concentrations $(0.5-50 \mathrm{vol} \%)$ and different flow types. Both authors concluded that the effect of microsized particles is qualitatively similar to an increased fluid viscosity of the dispersed phase, thereby resulting in a higher bulk viscosity ratio. Both studies used particles resulting in small particle over droplet size ratios $(r / R \sim 0.02$ 0.11). Mehrabian et al. (2015) on the other hand used higher particle over droplet size ratios $(r / R \sim 0.21-0.47)$ at high particle concentration ( $72 \mathrm{vol} \%$ ) and concluded by variation of the viscosity ratio $(\lambda=0.032-2.5)$ that breakup is in this case completely governed by the viscosity of the interstitial fluid of the dispersed phase.

From studies of the detachment of droplets from a needle, it is concluded that the droplet medium can no longer be considered as a continuum when a thin thread is formed or when concentration gradients exist due to particle rearrangements, (Furbank and Morris 2004; Bonnoit et al. 2012). Subsequently, different stages are identified during droplet detachment of a suspension. In the first stage of detachment, the system can still be treated as a continuum, hence the dynamics are similar to those of a pure fluid with an increased viscosity (Furbank and Morris 2004; Bertrand et al. 2012; Bonnoit et al. 2012). When the droplet is further detached, particle rearrangements in the filament start to create particle-free regions which dominate and accelerate the dynamics. Finally, close to complete detachment, localized thinning is responsible for accelerated droplet detachment (Bertrand et al. 2012; Bonnoit et al. 2012). These situations also typically occur during droplet breakup, for example during breakup of starch-filled droplets under shear flow (Desse et al. 2009). By rearrangement of the starch granules in shear flow, the droplet can easily break up at the depleted zone between two granules. However, the yield stress present in these highly concentrated particle-filled droplets also inhibits breakup completely for droplets with a viscosity ratio above 0.1 (Desse et al. 2009).

In general, some of the studies suggest that particle-filled droplets can be treated as droplets with a bulk viscosity higher than that of the droplet fluid. This bulk viscosity is governed by the particle concentration in the droplet. Consequently, particles are likely to reduce the droplet deformation and slow down the startup and retraction behavior. In addition, a stabilizing effect against droplet breakup is expected. However, a full quantitative study to investigate whether droplet dynamics of particle-filled droplets is indeed governed by the bulk viscosity and to explore possible additional particle effects is still lacking.

Additionally, it has to be noted that in most studies on dynamics of particle-filled droplets in shear flow, the added (nano)particles not only increase the droplet viscosity but also introduce non-Newtonian rheological behavior such as shear- 
thinning, viscoelasticity, and yielding (Desse et al. 2009; Desse et al. 2011; Kong et al. 2011; Gooneie et al. 2015). The observed changes in deformation and breakup dynamics are then associated with these non-Newtonian properties. However, non-Newtonian behavior makes it also difficult to compare different systems at similar capillary numbers or viscosity ratios (Desse et al. 2011).

The intrinsic effects of particles localized in the dispersed phase on the single droplet dynamics remain poorly understood to date. Therefore, we experimentally study the steady-state deformation, transient dynamics, and breakup behavior of single droplets containing microsized particles that do not introduce non-Newtonian properties. The results are systematically compared with the droplet dynamics of particle-free reference systems with comparable viscosity ratios. This will allow verifying the hypothesis that particlefilled droplets can be treated as a homogeneous medium characterized by its bulk viscosity which is governed by the particle concentration.

\section{Materials and methods}

\section{Materials}

Various grades of poly(dimethylsiloxane) (PDMS; Rhodorsil 47 V12500, Silbione 70047 V30000, Bluesil 47 V5000; Bluestar Silicones) are used as matrix phase in this study. Poly(ethylene glycol-ran-propylene glycol) (PEG-ran-PPG; $M_{\mathrm{n}} \sim 12,000$; Sigma Aldrich; batch MKBW7320V and MKBF0164V) is selected as droplet phase. The density of PDMS is $973 \mathrm{~kg} / \mathrm{m}^{3}$ at $20{ }^{\circ} \mathrm{C}$ (Minale et al. 1997) and the density of PEG-ran-PPG is $1092 \mathrm{~kg} / \mathrm{m}^{3}$ at $25^{\circ} \mathrm{C}$ (data from supplier). In order to obtain a specific desired viscosity ratio, PDMS mixtures are prepared according to the viscosity mixing rule described in Velankar and Moldenaers (2002). The viscosities of all fluids were measured with an AR-G2 rheometer (TA Instruments) equipped with a cone-plate setup. All components behave as Newtonian liquids in the shear rate range applied during the experiments $(0.1 / \mathrm{s}-$ 10/s).

Polystyrene particles (Dynoseeds; $\rho_{\mathrm{PS}}=1050 \mathrm{~kg} / \mathrm{m}^{3}$ at $20{ }^{\circ} \mathrm{C}$ (data from supplier)) are used to prepare the particlefilled droplets. The number average particle diameters are determined with optical microscopy and are equal to $25 \pm$ $2.7 \mu \mathrm{m}$ and $82 \pm 5.1 \mu \mathrm{m}$, which is in good agreement with the information given by the supplier (respectively, $23.1 \mu \mathrm{m}$ and $80.4 \mu \mathrm{m}$ ). This method was not applicable for the smallest particles; hence, a particle size of $5.3 \pm 0.093 \mu \mathrm{m}$ as given by the supplier is used for these particles.

Suspensions are prepared by adding the particles to PEGran-PPG (batch MKBW7320V; $\eta=37.4 \mathrm{~Pa}$ s at $23^{\circ} \mathrm{C}$ ) and mixing with a spatula for 10 min. Hereafter, entrapped air bubbles are removed by keeping the suspension overnight in a vacuum oven at room temperature. Suspensions with a particle concentration of 5, 10, 15, and $20 \mathrm{vol} \%$ are prepared and used as droplet phase. The creaming of particles in the bulk suspensions can be neglected regarding the timeframe of the rheology and droplet dynamics experiments as demonstrated in Supplementary information. To verify that the suspensions behave as purely Newtonian liquids in the shear rate range of interest, their rheological behavior is characterized by performing a strain sweep and a frequency sweep in the linear viscoelastic region as well as a steady-state flow sweep. These experiments are performed with a parallel plate setup $(D=$ $40 \mathrm{~mm}$, gap height $=1 \mathrm{~mm}$ ). The viscosity is indeed independent of the shear rate in the shear rate range of interest $(0.01 / \mathrm{s}$ $10 / \mathrm{s})$. Moreover, $G$ " linearly increases with angular frequency and $G$ ' is negligibly small, thereby indicating negligible elasticity.

Figure 1 displays the relative viscosity $\eta_{\mathrm{r}}$ of the suspensions as a function of their particle concentration $\varphi$. The relative viscosity is defined as the ratio of the bulk suspension viscosity over the viscosity of the suspending medium, being PEGran-PPG (batch MKBW7320V). As expected, the suspension viscosity only depends on the particle concentration and is independent of the particle size. The full line in Fig. 1 represents the Maron-Pierce equation (Mewis and Wagner 2011), with 0.64 as maximum packing fraction $\left(\varphi_{\mathrm{m}}\right)$ :

$\eta_{\mathrm{r}}=\left(1-\frac{\varphi}{\varphi_{\mathrm{m}}}\right)^{-2}$

This model is a good fit for the measured data and, hence, can be used to estimate the viscosity of suspensions with intermediate particle concentrations.

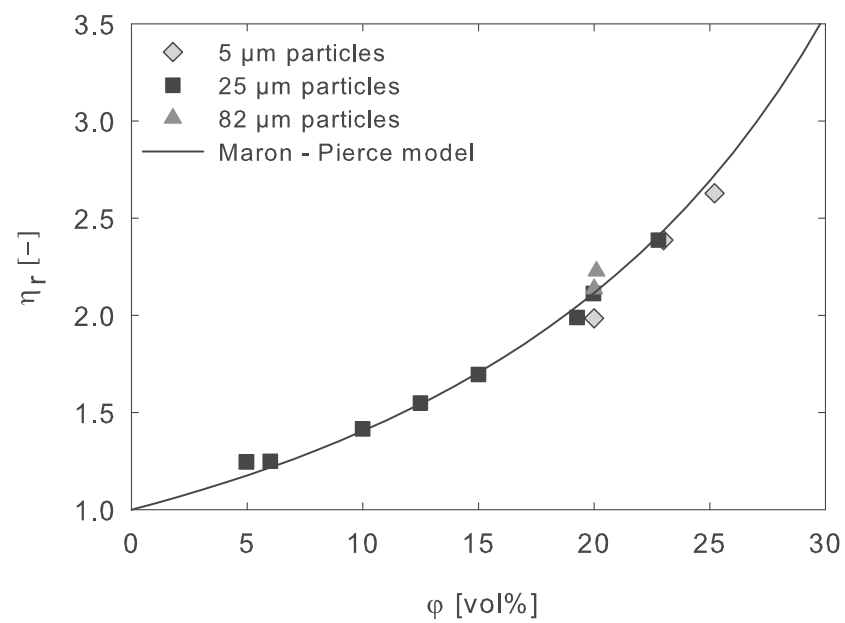

Fig. 1 Relative viscosity $\eta_{\mathrm{r}}$ of bulk PEG-ran-PPG suspensions with 5 $\mu \mathrm{m}, 25 \mu \mathrm{m}$ and $82 \mu \mathrm{m}$ sized particles as function of particle concentration at $23{ }^{\circ} \mathrm{C}$. The full line indicates the Maron-Pierce equation (Eq. 1) with 0.64 as maximum packing fraction 


\section{Particle systems and reference systems}

To study the effects of particles on the single droplet dynamics, experiments are performed on different particle systems (PSX-Y, with $\mathrm{X}$ as the particle size and $\mathrm{Y}$ as the particle concentration in vol\%). Table 1 summarizes the main characteristics of these particle systems. All particle systems consist of a PDMS matrix phase with a viscosity of $28.7 \mathrm{~Pa} \mathrm{~s}\left(23^{\circ} \mathrm{C}\right)$.

The particle concentrations of the injected droplets differ significantly from the concentrations of the prepared suspensions due to the formation of some small particle-free satellite droplets during injection. To determine the particle concentration of the droplets, an average of at least 8 single droplets is investigated. First, the droplet size is determined and thereafter, the single droplet is transferred with a spatula to a microscope slide. Subsequently, the droplet is carefully squeezed between two microscope slides which make it possible to count the number of particles present in the droplet. Hence, with the particle size, droplet size, and number of particles known, the particle concentration in the droplet can be calculated. However, this method is not applicable for the system which contains 5- $\mu \mathrm{m}$-sized particles (PS5-25) because the particles are too small to individually count them at a magnification that allows seeing the complete droplet in the field of view. For this reason, the particle concentration of this system is determined by using confocal microscopy. Droplets containing $20 \mathrm{vol} \%$ fluorescent silica particles with a similar particle size $(7 \mu \mathrm{m})$ as system PS5-25 are injected into PDMS and scanned in 3D to determine the particle volume fraction with image analysis. We assume that the determined particle volume fraction of the droplets filled with fluorescent silica particles is similar to that of the droplets containing polystyrene particles with a similar size.

The bulk viscosity of the particle-filled droplets is then determined by measuring the viscosity of a suspension with a particle concentration equal to the average particle concentration of the droplets. These data are included in Fig. 1. Finally, the bulk viscosity ratio $\lambda$ is determined and listed in Table 1. In all further figures, this value of the viscosity ratio is used. In Table 1, we also estimated a corresponding droplet viscosity for the observed minimal and maximal particle concentrations with the Maron-Pierce model (Mewis and Wagner 2011), indicated by the full line in Fig. 1.

The interfacial tension $\Gamma$ is determined by fitting the droplet axes at small deformations to the second order theory of Greco (2002). Table 1 shows the average interfacial tension of at least three experiments. However, the interfacial tension is determined separately for every investigated droplet in systems for which deviations larger than 5\% were observed. By comparison of the interfacial tension values of the particle systems in Table 1 with those of the particle-free reference systems $(8.5 \mathrm{mN} / \mathrm{m}$, see Table 2$)$, it becomes clear that the particles do not significantly alter the interfacial tension.

The droplet dynamics of particle systems (PS) and particlefree reference systems with comparable viscosity ratios are compared in this work. These reference systems consist of a PEG-ran-PPG droplet (batch MKBF0164V, $\eta=$ $40.0 \mathrm{~Pa} \mathrm{~s}$ at $23{ }^{\circ} \mathrm{C}$ ) and a PDMS matrix phase. Table 2 shows the matrix viscosities and resulting viscosity ratios of the different reference systems RS-Z with $\mathrm{Z}$ the viscosity ratio. The matrix and droplet phase of reference system RS-1.4 consist of exactly the same grades as those of the particle systems, while the matrix viscosity of the other reference systems is adjusted in order to match the viscosity ratio with that of a specific particle system.

The interfacial tension of RS-1.4, determined by fitting small droplet deformations with the second order theory of Greco (2002), is equal to $8.5 \pm 0.07 \mathrm{mN} / \mathrm{m}$. The interfacial tension is independent of the molecular weight of PDMS for the used molecular weight (Kobayashi and Owen 1995; Vananroye et al. 2007), and hence, the value of $8.5 \mathrm{mN} / \mathrm{m}$ is also used for the other reference systems.

\section{Experimental setup and protocol}

Experiments are conducted using a counter-rotating parallel plate shear cell. Figure 2 shows a sketch of the parallel plate shear cell, more details about this setup can be found elsewhere (Vananroye et al. 2006; Verhulst et al. 2007). Images of the droplet, kept in the stagnation plane, are captured with a
Table 1 Characteristics of the particle systems (PS): average particle volume concentrations $( \pm$ standard deviation), viscosity ratios $\lambda$ at a temperature of $23^{\circ} \mathrm{C}$, range of viscosity ratios resulting from the variation in particle concentration and average interfacial tensions ( \pm standard deviation)

\begin{tabular}{llllr}
\hline Particle system & Particle concentrations [vol\%] & $\lambda[-]$ & Range $\lambda[-]^{\mathrm{a}}$ & $\Gamma[\mathrm{mN} / \mathrm{m}]$ \\
\hline PS25-6 & $6.0 \pm 1.0$ & 1.6 & $1.5-1.7$ & $8.0 \pm 0.11$ \\
PS25-13 & $12.5 \pm 2.3$ & 2.0 & $1.8-2.2$ & $8.2 \pm 0.08$ \\
PS25-19 & $19.3 \pm 2.2$ & 2.6 & $2.3-3.1$ & $8.3 \pm 0.16$ \\
PS25-23 & $22.8 \pm 1.5$ & 3.1 & $2.8-3.5$ & $8.6 \pm 0.16$ \\
PS82-20 & $20.1 \pm 0.3$ & 2.9 & $2.7-2.8$ & $8.3 \pm 0.12$ \\
PS5-25 & $25.2 \pm 1.4$ & 3.4 & $3.1-3.8$ & $8.9 \pm 0.07$ \\
\hline
\end{tabular}

${ }^{\text {a }}$ Range is estimated using the Maron-Pierce equation (Eq. 1) and the minimal and maximal observed particle volume concentrations 
Table 2 Newtonian matrix viscosities and resulting viscosity ratios of the particle-free reference systems (RS) at a temperature of $23{ }^{\circ} \mathrm{C}$

\begin{tabular}{|c|c|c|c|}
\hline Reference system & $\eta_{\mathrm{m}} \quad[\mathrm{Pa} \mathrm{s}]$ & $\lambda[-]$ & $\Gamma[\mathrm{mN} / \mathrm{m}]$ \\
\hline RS - 1.4 & 28.7 & 1.4 & \\
\hline RS -1.7 & 23.1 & 1.7 & \\
\hline RS -2.2 & 18.5 & 2.2 & $8.5 \pm 0.07$ \\
\hline RS -3.2 & 12.6 & 3.2 & \\
\hline RS - 3.4 & 11.9 & 3.4 & \\
\hline
\end{tabular}

microscope and camera in two planes: the velocity-vorticity plane (top view) and the velocity-velocity gradient plane (side view). The droplet axes and orientation angle $\theta$ of the deformed droplet, as indicated in Fig. 2, can be determined from these images by assuming an ellipsoidal droplet shape.

In this work, the droplet radius $(R)$ is equal to $109 \pm 3 \mu \mathrm{m}$ and the gap height $(H)$ is set to $3 \mathrm{~mm}$. As shown in Supplementary information, gravitational effects can be neglected regarding the time frame of the experiments. In addition, the vertical position of the droplet is always checked to ensure that wall effects can be neglected. The temperature of the sample is determined with a thermocouple for every experiment. This temperature is used to calculate the viscosity ratios of the data shown in the results section. The experimental temperature is equal to $23.2 \pm 0.3{ }^{\circ} \mathrm{C}$.

The random particle distribution in the droplet after injection is always verified with microscopy images (top and side

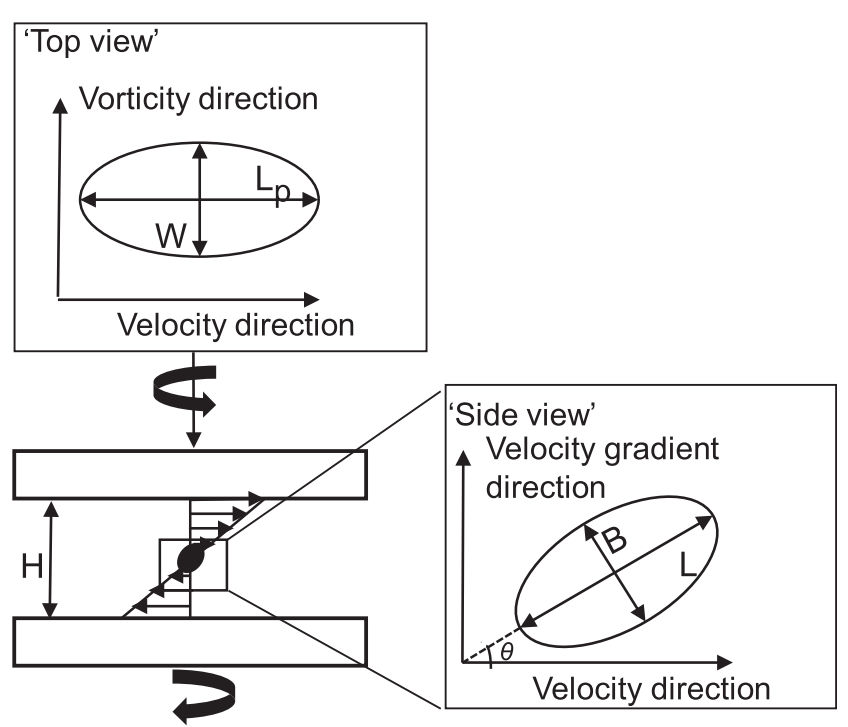

Fig. 2 Sketch of parallel plate shear cell in side view with the vorticity direction perpendicular to the plane. The camera position to capture images in top view is indicated with an arrow. The droplet axes $L, B, W$ and $L_{p}$ and orientation angle $\theta$ are indicated on the schematic of the deformed droplet in top and side view. view). It is verified that the particles do not migrate to the interface nor to the matrix phase when the droplet is kept under quiescent conditions. However, during the application of shear, the particles migrate towards the central droplet axis in the top view. The nonhomogenous steady-state particle distribution is independent of the applied shear rate, an example can be found in Figs. S1a-b of the Supplementary information. The systematic investigation and discussion of the kinetics of this particle migration will be the subject of another work, currently under preparation. For the present work, it is verified that the particle distribution has no significant effect on the droplet dynamics, an example can be found in Supplementary information.

The following measurement protocol is applied. For every droplet, the steady-state droplet deformation at $\mathrm{Ca}=0.30$ is first determined immediately after droplet injection and, hence, with a homogeneous particle distribution. For system PS25-23, the steady-state droplet deformation as function of capillary number is also determined. Subsequently, the transient startup and retraction behavior of this droplet is observed at capillary numbers of 0.2 and 0.3 . Finally, the breakup behavior is studied in more detail by stepwise increasing the applied capillary number until droplet breakup occurs. The transient and breakup experiments are performed with a nonhomogeneous steady-state particle distribution. To ensure this steady-state particle distribution, a long period of shear flow (5-10 min. at $\mathrm{Ca}=0.30)$ is applied between the deformation experiment and the subsequent transient and breakup experiments. Every capillary number is applied for maximum $5 \mathrm{~min}$. Hereafter, the shear flow is stopped, and hence, the droplet retracts back to a spherical shape, before applying the next capillary number.

\section{Results}

This section describes the steady-state deformation, startup, retraction, and breakup behavior of the particle systems. We 
expect to observe a reduced droplet deformation, slower transient dynamics, and droplet breakup at higher critical capillary numbers compared with RS-1.4 as a consequence of the increased viscosity ratio. The droplet dynamics are also compared with those of reference systems with a comparable viscosity ratio in order to verify the hypothesis that particle-filled droplets behave as a homogeneous medium characterized by the bulk viscosity of the suspension. Therefore, every section first compares the behavior of PS25-23 with RS-1.4, RS-3.2, and predictions of the Maffettone-Minale (MM) model (Maffettone and Minale 1998). Hereafter, the effect of particle over droplet size ratio and particle concentration is discussed in a summarizing graph of a characteristic parameter for the specific experiment as function of the bulk viscosity ratio.

\section{Steady-state deformation and orientation}

Figure 3 compares the dimensionless droplet axes $(L / 2 R, W / 2 R$, and $B / 2 R$ ) and orientation angles $\theta$ of deformed droplets of PS2523 as function of capillary number with those of the particle-free reference systems RS-1.4 and RS-3.2. In addition, predictions of
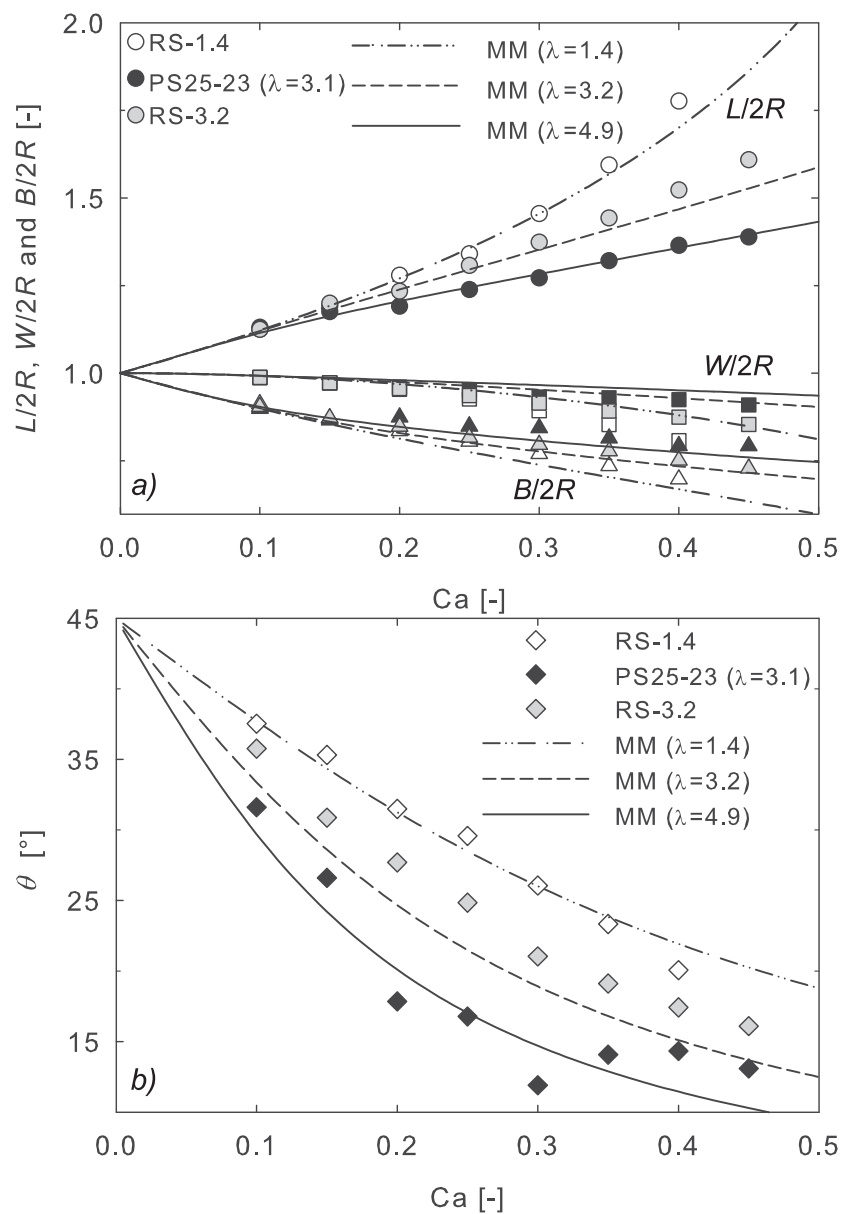

Fig. 3 (a) Dimensionless droplet axes $L / 2 R, W / 2 R$ and $B / 2 R$ and (b) Orientation angles $\theta\left[^{\circ}\right]$ as function of capillary number $\mathrm{Ca}$. the MM model (Maffettone and Minale 1998) are shown as dashed lines in Fig. 3.

As expected, Fig. 3a shows good agreement (deviations $<$ $5 \%$ ) of $L / 2 R$ with the corresponding MM model up to relatively high capillary numbers for RS-1.4 and RS-3.2. In addition, the model is able to accurately predict the droplet orientation of systems with a viscosity ratio around one, as can be seen in Fig. 3b for RS-1.4. However, for viscosity ratios much higher than one (RS-3.2), the model predicts more orientation towards the flow direction than experimentally observed (Maffettone and Minale 1998; Vananroye et al. 2007).

It is clear from Fig. 3 that the observed steady-state deformation of PS25-23 does not correspond to that of RS-1.4. The particle-filled droplets of PS25-23 deform less and orient more towards the flow direction compared with RS-1.4. A reduced deformation is also observed experimentally in nanoparticle-filled blends (Gooneie et al. 2015) and in numerical simulations (Usta et al. 2009). The addition of (nano)particles introduces additional viscous and/or elastic stresses resisting droplet deformation (Usta et al. 2009).

The data for PS25-23 in Fig. 3 are also not in agreement with the data of the corresponding particle-free system with the same viscosity ratio (RS-3.2). The deviations between particle system PS25-23 and RS-3.2 are relatively small $(\leq$ $7 \%)$ at low and intermediate capillary numbers $(\mathrm{Ca} \leq 0.30)$, but become more pronounced at higher capillary numbers $(14 \%$ at $\mathrm{Ca}=0.45)$. The MM model is fitted through the $L / 2 R$ data of PS25-23 with the viscosity ratio $\lambda$ as fitting parameter. The resulting fit $(\lambda=4.9)$ is also depicted in Fig. 3 as a full line. According to the MM model, the steady-state deformation of system PS25-23 is thus similar to the steady-state deformation of a particle-free droplet with a viscosity ratio of 4.9 , which is higher than the bulk viscosity ratio of this system $(\lambda=3.1)$.

The effect of particle concentration and particle size on the steady-state droplet deformation is summarized in Fig. 4, which gives an overview of $L / 2 R$ at $\mathrm{Ca}=0.30$ as function of the bulk viscosity ratio $\lambda$. For every reference system, three droplets with similar parameters are measured and indicated as separate data points in Fig. 4. Analogously, experiments with 3-5 droplets are performed for the particle systems because these particle systems are less reproducible than the reference systems, but differences between different droplets remain smaller than $5.5 \%$. Repeated measurements on the same droplet show a deviation in $L_{\mathrm{p}} / 2 R$ of less than $4 \%$. It has to be noted that Fig. 4 is a detailed figure compared with Fig. 3 a as the $L / 2 R$-axis in Fig. 4 only spans a very small range $(1.20-1.50)$. All measured values of the reference systems (RS) are slightly larger than the MM model predictions, although deviations are small $(<2.5 \%)$.

The effect of particle concentration is studied by means of the systems PS25-Y in Fig. 4. Figure 3 already demonstrated that PS25-23 behaves as a system with a viscosity ratio higher than its bulk viscosity ratio. Figure 4 confirms this trend for 


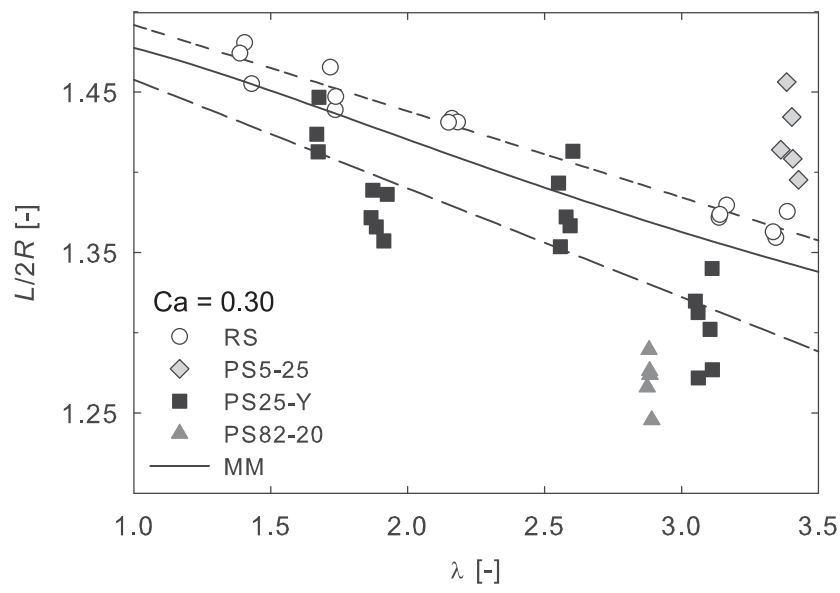

Fig. 4 Dimensionless droplet axis $L / 2 R$ as function of the bulk viscosity ratio $\lambda$. Data points with approximately the same viscosity ratio represent different droplets of the same particle or reference system. Linear fits through the data points of RS and PS25-Y are shown by dashed lines to guide the eye. The full line indicates the MM model prediction. All data are for $\mathrm{Ca}=0.30$

other particle concentrations as most particle-filled droplets of PS25-Y show a reduced deformation compared with droplets from the corresponding reference system. This effect increases with increasing particle concentration but remains relatively small for a capillary number of 0.30 . Deviations of PS25-23 from RS-3.2 range from 2 to $7 \%(\mathrm{Ca}=0.30)$. However, Fig. 3 already illustrated that this effect becomes more pronounced at higher capillary numbers.

Figure 4 also illustrates the effect of particle size. Similar to PS25-23, also PS82-20 shows a reduced dimensionless droplet axis $L / 2 R$. This reduction is even more pronounced for PS82-20 than for PS25-23, which shows that the particle effect depends on particle size. On the other hand, PS5-25 has an average deformation even slightly larger (2.4-6.8\%) than the reference system with the same viscosity ratio, but still smaller or equal to the original reference system RS-1.4 (deviations: $0-6 \%$ ).

An additional particle effect observed during steady-state deformation is illustrated in Fig. 5. Figure 5a shows the dimensionless parameter $L_{\mathrm{p}} / 2 R$ after startup of shear flow at $\mathrm{Ca}=0.30$, followed by a period of steady shear flow. $L_{\mathrm{p}}$ represents the projection of the droplet axis $L$ in the top view (see Figs. 2 and $5 \mathrm{c}$ ). Time is made dimensionless by dividing it with the respective emulsion time $\tau_{\mathrm{E}}\left(=\eta_{\mathrm{m}} R / \Gamma\right)$. The startup dynamics will be discussed in detail in the next section, here we focus on the steady-state behavior after startup $\left(t / \tau_{\mathrm{E}}>20\right)$.

As expected, the reference systems (RS) have a stable ellipsoidal droplet shape during steady shear flow and hence, also constant values of $L_{\mathrm{p}} / 2 R$. As illustrated in Fig. 5a, PS5-25 behaves in a similar way. This is in contrast to systems PS25-23 and PS82-20, which clearly show a small overshoot in $L_{\mathrm{p}} / 2 R$ after startup of shear flow followed by small-amplitude oscillations $\left(\left(L_{\mathrm{p}, \max }-L_{\mathrm{p}, \min }\right) / L_{\mathrm{p}, \mathrm{ss}} \leq 1.1 \%\right)$. The oscillations observed in
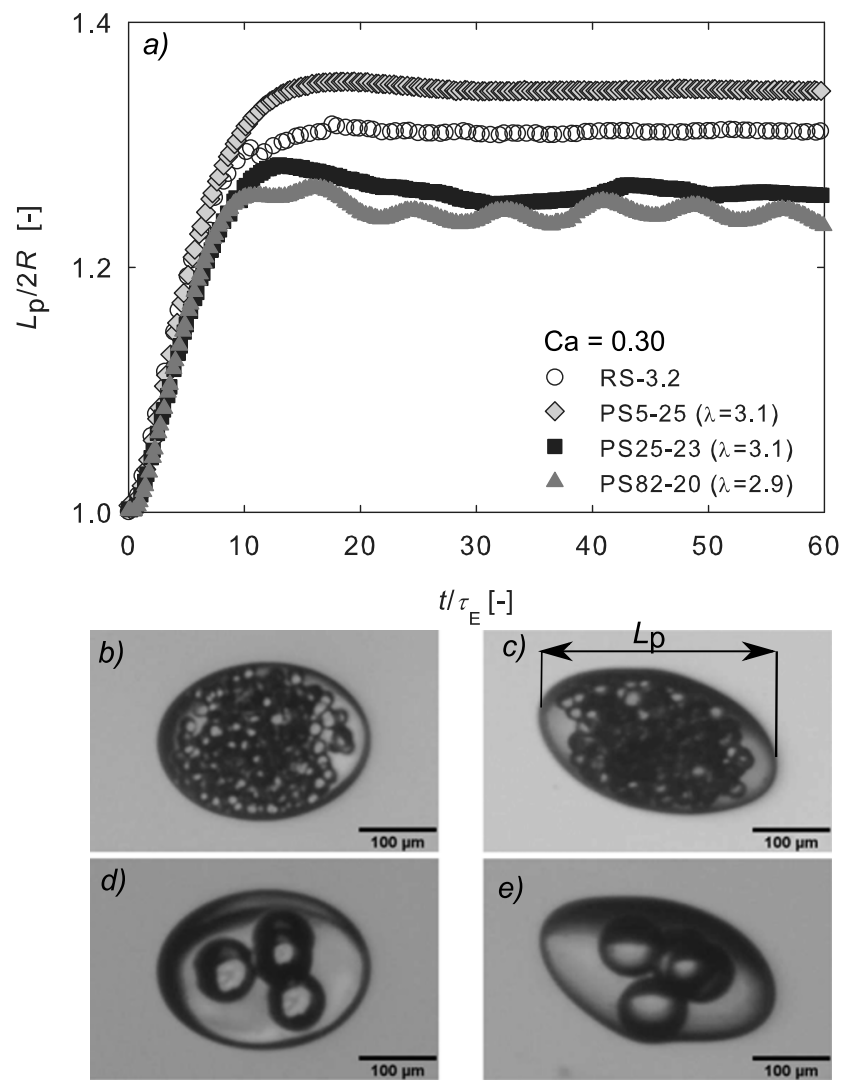

Fig. 5 (a) Dimensionless parameter $L_{p} / 2 R$ as function of dimensionless time $t / \tau_{\mathrm{E}}$ after startup of shear flow. Images of PS25-23 (b-c) and PS82-20 (d-e) during steady-state shear. Images are taken in the top view (b-d) and the side view (c-e). All data are for $\mathrm{Ca}=0.30$

our experiments are still present after several minutes of shearing. For the same particle system, it was qualitatively observed that the time period of the oscillations decreases with increasing capillary number and the fluctuations become more pronounced at higher capillary numbers, higher particle concentrations, or higher particle over droplet size ratios. A similar phenomenon has been reported for droplets submersed in a suspension in which the particles in the matrix phase cause local flow disturbances (Kaur and Leal 2010). An overshoot during startup of shear flow is also observed by Smith and Van de Ven (1985a) for a droplet containing a single particle.

In general, the rotational flow inside the droplet causes the particles to circulate. At high particle concentrations and high particle over droplet size ratios, particle confinement during this circulation results in outwards bulging of the particles. This also results in considerable deviations from the assumed ellipsoidal droplet shape as can be seen in Supplementary video ESM 1 and Fig. 5b-e. Desse et al. (2009) also mentioned irregularities in droplet shape when starch granules are added to water in oil droplet. These irregularities are attributed to the internal circulation of the granules inside the droplet. For system PS82-20, the oscillations are caused by individual particles bulging out of the droplet, while for system PS25-23, 
the irregular shape of the large particle cluster is responsible for the oscillations and deviations in droplet shape. Consequently, the oscillations observed for PS82-20 have a larger amplitude and smaller time period. However, considering the limited amplitude of the oscillations and lack of regularity, a full quantitative analysis of these oscillations is considered beyond the scope of the experiments.

\section{Transient droplet deformation after startup of shear flow}

In Fig. 6a, the startup curves of reference systems RS-1.4, RS3.2, and PS25-23 are compared with each other and with the corresponding predictions of the MM model. In this figure, the time evolution of the dimensionless parameter $\left(\left(L_{\mathrm{p}} / 2 R\right)-1\right)$ is shown from the moment shear flow is started until a steadystate deformation is reached. However, as discussed in the
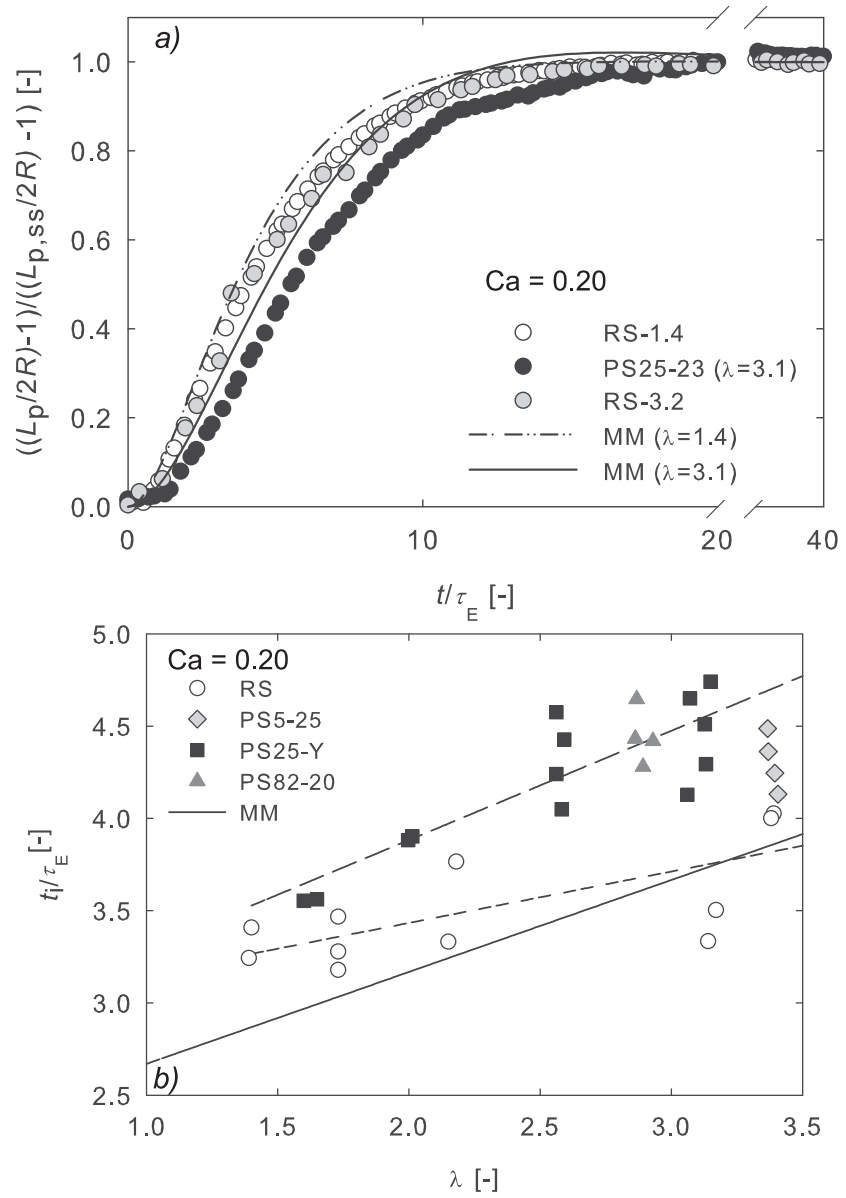

Fig. 6 (a) Dimensionless parameter $\left(\left(L_{p} / 2 \mathrm{R}\right)-1\right)$ normalized by its steadystate value $\left(\left(L_{p, s s} / 2 R\right)-1\right)$ as function of dimensionless time $t / \tau_{\mathrm{E}}$ during startup of shear flow (b) Dimensionless startup time $t_{\mathrm{i}} / \tau_{\mathrm{E}}$ as function of the bulk viscosity ratio $\lambda$. Data points with approximately the same viscosity ratio represent different droplets of the same particle or reference system. Linear fits through the data points of RS and PS25-Y are shown by dashed lines to guide the eye. The full line indicates the MM model prediction. All data are for $\mathrm{Ca}=0.20$ previous section, the systems attain different steady-state values for the droplet axes. To be able to adequately compare the startup dynamics of the different systems, we therefore normalize $\left(\left(L_{\mathrm{p}} / 2 R\right)-1\right)$ by its steady-state value $\left(\left(L_{\mathrm{p}, \mathrm{ss}} / 2 R\right)-1\right)$. In addition, time is made dimensionless by dividing it with the respective emulsion time $\tau_{\mathrm{E}}$.

Differences between the startup curves in Fig. 6a remain limited, and the overall shapes are similar. RS-1.4 reaches its steady-state deformation somewhat later than expected from the MM model prediction, while the MM model prediction for the high viscosity ratio system (RS-3.2) is in good agreement with the experimental data.

The startup curve measured for PS25-23 clearly deviates from the curves measured for the reference systems. The particle system reaches its steady-state value at a later time; hence, it is characterized by slower startup dynamics. This result is similar to the findings of Gooneie et al. (2015) who reported a decreased droplet deformation rate when carbon nanotubes are added to a PP/PA6 blend and hinder the mobility of the dispersed phase.

To be able to compare the different systems in a more quantitative way, a characteristic time scale for the startup curves is determined. All startup curves at $\mathrm{Ca}=0.20$ have a sigmoidal shape which is very similar to the shape of the Gompertz function (Tjørve and Tjrøve 2017):

$y(t)=A e^{-e^{-\left(k\left(t-t_{\mathrm{i}}\right)\right)}}$

in which $k$ is the slope at the inflection point, $t_{\mathrm{i}}$ the time at inflection, and A the upper asymptote, which is in our case equal to one. A two-parameter fit of Eq. 2 is made through all the startup curves, including the MM model predictions and $t_{\mathrm{i}}$ is selected as the characteristic time scale for the startup process. The resulting values of $k$ are also compared, but no significant differences or clear trends could be observed. A summary of the resulting $t_{\mathrm{i}}$ values for the different systems can be found in Fig. 6b. Note that the characteristic time scale $t_{\mathrm{i}}$ is made dimensionless with the corresponding emulsion time $\tau_{\mathrm{E}}$.

The characteristic time scales calculated from the experimentally determined curves of the reference systems do not always agree with the MM model prediction, approximated by the full line in Fig. 6b. Therefore, caution is recommended during the interpretation of these results. Additionally, there exists quite some variability on the fitted $t_{\mathrm{i}}$ values, yet some general conclusions can be made.

The characteristic time scales of all particle systems are larger than those of the corresponding reference systems. This confirms the conclusion, already made from Fig. 6a for system PS25-23, that the startup dynamics are slowed down by the addition of particles, more than could be expected on the basis of the bulk viscosity ratio. This effect increases with particle concentration. 
Surprisingly, the characteristic time scales for all systems containing 20 vol\% particles are approximately in the same range and thus independent of particle size. Hence, the kinetics towards steady-state are almost independent of the particle over droplet size ratio, while the reduction in steady-state deformation increases significantly with this ratio.

\section{Droplet retraction after cessation of shear flow}

Figure 7a illustrates the shape retraction of reference systems RS-1.4, RS-3.2, and particle system PS25-23, represented as the time evolution of $\left(\left(L_{\mathrm{p}} / 2 R\right)-1\right)$ normalized by its steadystate value during shear flow $\left(\left(L_{\mathrm{p}, \mathrm{ss}} / 2 R\right)-1\right)$. Time is made dimensionless by dividing it with the respective emulsion time $\tau_{\mathrm{E}}$, and time zero corresponds to the moment shear flow is stopped. The flow is stopped when a steady-state droplet deformation is reached. All retraction experiments are performed
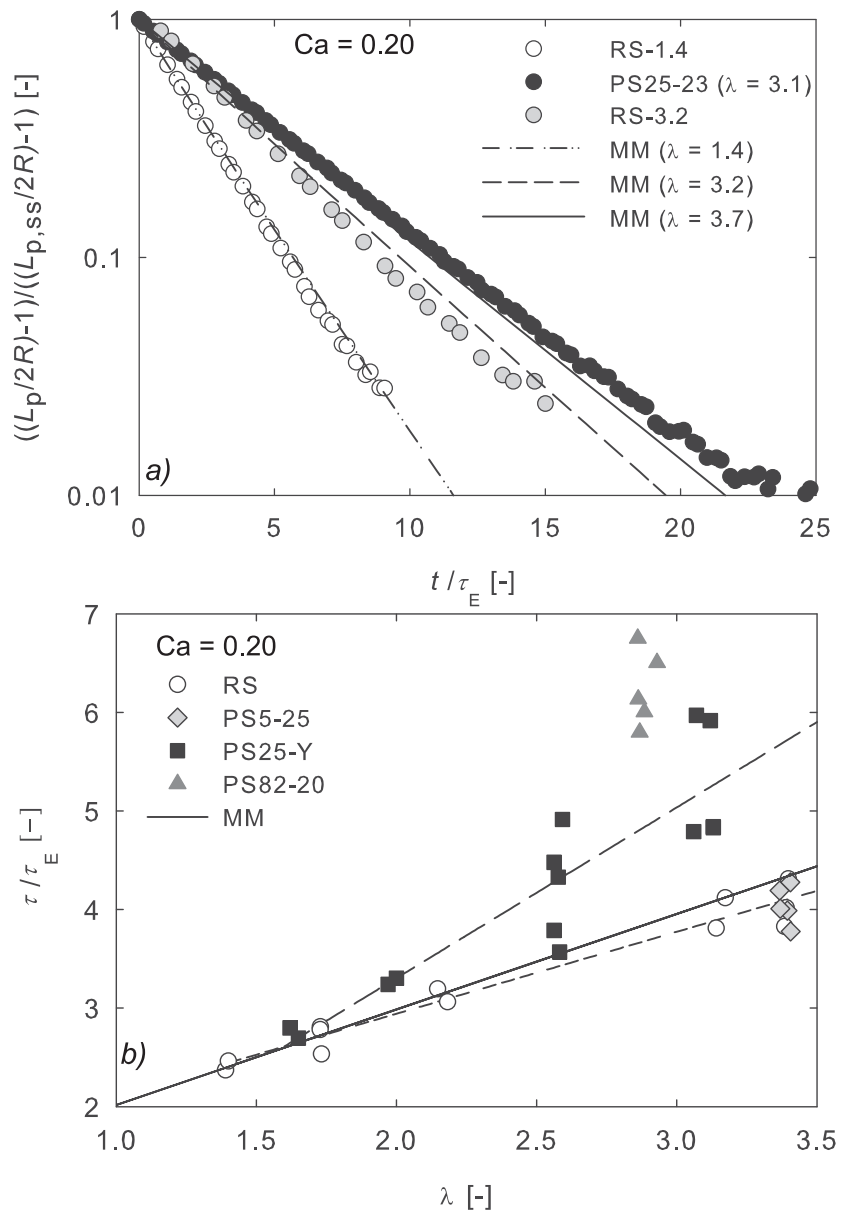

Fig. 7 (a) Dimensionless parameter $\left(\left(L_{p} / 2 R\right)-1\right)$ normalized by its steadystate value $\left(\left(L_{\mathrm{p}, \mathrm{ss}} / 2 R\right)-1\right)$ as function of dimensionless time $t / \tau_{\mathrm{E}}$ during droplet retraction (b) Dimensionless relaxation time $\tau / \tau_{\mathrm{E}}$ as function of bulk viscosity ratio $\lambda$. Data points with approximately the same viscosity ratio represent different droplets of the same particle or reference system. Linear fits through the data points of RS and PS25-Y are shown by dashed lines to guide the eye. The full line indicates the MM model prediction. All data are for $\mathrm{Ca}=0.20$ after applying a shear flow corresponding to $\mathrm{Ca}=0.20$. As expected, the retraction curves of RS-1.4 and RS-3.2 correspond well with the MM model predictions.

On the other hand, PS25-23 retracts significantly slower as compared with the corresponding reference system with similar viscosity ratio, namely RS-3.2. Thus, PS25-23 retracts in a similar way as a particle-free system with a higher viscosity ratio than its bulk viscosity ratio. The PA6/PS system studied by Kong et al. (2011) also showed slower retraction dynamics of single droplets containing silica nanoparticles. In contrast, Gooneie et al. (2016) found an accelerated retraction process of PA6 droplets filled with carbon nanotubes which form an elastic network. However, the particle-filled blends exhibit in both cases viscoelastic behavior which is not the case in our study. In addition, both studies only compared the retraction behavior of the particle-filled droplets with those of the neat blend which has different viscous and elastic properties.

From each retraction curve in Fig. 7a, a single characteristic relaxation time scale $\tau$ can be calculated by fitting an exponential function $\left(\mathrm{e}^{-t / \tau}\right)$. Figure $7 \mathrm{~b}$ provides a summary of these relaxation times, scaled by the respective emulsion time $\tau_{\mathrm{E}}$, as function of the bulk viscosity ratio $\lambda$. The qualitative trend observed in Fig. 7a is confirmed by this overview.

The experimentally determined relaxation times of the reference systems agree well with the MM model predictions, while particle systems PS25-Y and PS82-20 show longer relaxation times when the particle concentration is $10 \mathrm{vol} \%$ or higher. Hence, these particle-filled droplets retract slower and thus behave similar to systems with a higher viscosity ratio than their bulk viscosity ratio. This effect becomes again more pronounced at higher particle concentrations and higher particle to droplet size ratios. In contrast, the relaxation times of PS5-25 are in good agreement with those of the respective reference system (RS-3.4). At this low particle to droplet size ratio, the particle suspension that constitutes the droplet can thus be considered as a homogeneous medium characterized by the bulk viscosity ratio governed by the particle concentration.

It has to be noted that significant deviations (13$38 \%$ ) in relaxation time are observed between different experiments with droplets containing 15 vol\% or more particles. However, different retraction experiments at $\mathrm{Ca}=0.20$ and $\mathrm{Ca}=0.30$ performed with the same droplet of PS25-Y resulted in one retraction master curve, and hence a reproducible value (deviations $<10 \%$ ) of the relaxation time per droplet. This suggests that the variability in relaxation time is caused by variations in particle concentration between different droplets. To verify this hypothesis, the particle concentration of different droplets of PS25-19 is determined after the performed experiments, using the method explained in the "Materials and methods" section. The bulk viscosity ratio of that particular system is then calculated with the 
Maron-Pierce equation (Mewis and Wagner 2011). However, as illustrated in Supplementary information, the trend with viscosity ratio does not become clearer when the results are plotted as a function of the calculated viscosity ratio per droplet instead of the average viscosity ratio as tabulated in Table 1 . Hence, the variation in particle concentration can only partially explain the increase in variability for concentrations above 15 vol\%. Probably, local intrinsic particle effects cause an additional variation in droplet behavior.

On the other hand, for system PS82-20, experiments at different capillary numbers on the same droplet already give rise to slightly different retraction curves. From these results, we can assume that at high particle over droplet size ratios (PS82-20), droplet retraction is affected by local particle effects which cause an increase in variability. This interpretation is supported by the deviations in droplet shape and resulting oscillations observed during steadystate shear flow in Fig. 5, which could be linked to the particle circulation inside the droplet.

\section{Droplet breakup}

Droplet breakup is studied by increasing the applied capillary number with small discrete steps until breakup occurs. Thereby, after every applied capillary number, the shear flow is stopped and, hence, the droplet starts from a spherical shape. Figure 8 provides an overview of the observed breakup mechanisms, illustrated by images taken at $\mathrm{Ca}_{\text {crit }}$ in the top view.

As expected, all reference systems break up by necking, which results in two identical daughter droplets and some satellite droplets. This is illustrated in Fig. 8 for system RS-1.4. The breakup modes observed for PS5-25, PS25-6, and some droplets of PS25-13 are similar to those of the reference systems. However, satellite droplets of PS25-6 and PS25-13 do not contain any particles, while satellite droplets of PS5-25 are partially filled with particles. For larger particles, the neck width during thinning becomes comparable with the particle size, and particles are forced towards the larger daughter droplets. Hence, a particlefree neck is created where droplet breakup occurs. Similar behavior is observed by Furbank and Morris (2004) during droplet formation of concentrated suspensions from a needle $(\geq$ $15 \mathrm{vol} \%$ ), yet with a lower particle over droplet size ratio.

On the other hand, PS25-19, PS25-23, PS82-20, and some droplets of PS25-13 show a completely different breakup behavior. As can be seen in Fig. 8 and in Supplementary video ESM 2, the particle-filled droplets do not break into two identical daughter droplets, instead particle-free droplets are released from the droplet tips. Attempts to break the remaining particle cluster by immediately applying a very high shear rate only resulted in the release of more and smaller particle-free satellite droplets from the tips. In blends and emulsions, this different breakup mechanism may lead to a broader droplet size distribution.

This breakup mechanism in Fig. 8 is comparable with tip streaming which is observed for systems with an interfacial tension gradient (de Bruijn 1993), although the physical origin is different. During shear flow, no particles are present in the droplet tips due to the particle clustering around the droplet center. As a result, a lower viscosity ratio, which is comparable with the viscosity ratio of RS-1.4, exists locally in the droplet tips. This lower viscosity ratio gives rise to easier
Fig. 8 Images of RS-1.4, PS5-25, PS25-Y and PS82-20 taken during droplet breakup at $\mathrm{Ca}_{\text {crit }}$ (indicated in the first column) in top view. Second and third column show images taken at time $t$ / $t_{\mathrm{b}}=0.2$ and $t / t_{\mathrm{b}}=0.7$, respectively, with $t_{\mathrm{b}}$ the breakup time. Last column shows the droplets just after breakup $\left(t / t_{\mathrm{b}}=1\right)$. The symbols in the first column refer to the symbols used in Figures 9 and 10

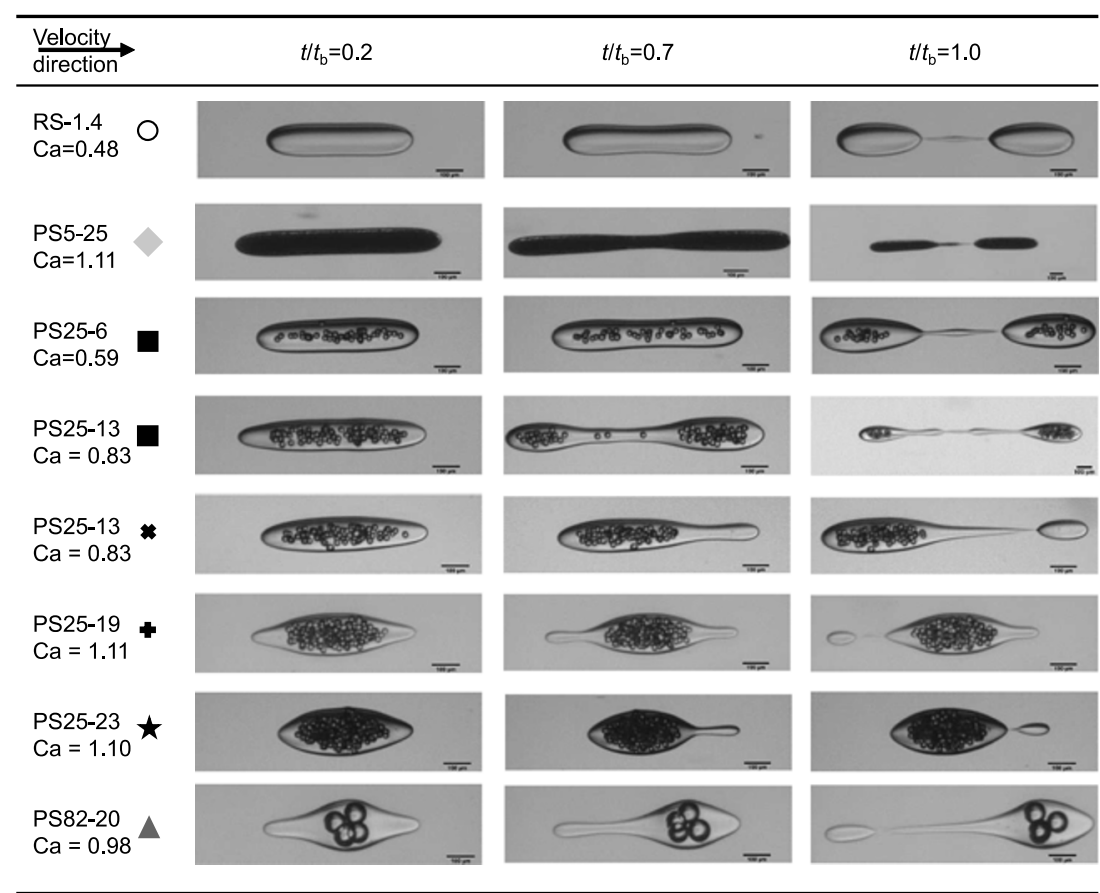


breakup of the particle-free droplet tips as compared with the particle-filled cluster in the droplet center. Consequently, only satellite droplets without particles are formed. As a result, the particle concentration of the remaining particle cluster is higher than the particle concentration of the original droplet. This effect stabilizes the droplet against further breakup (Powell and Mason 1982). The opposite behavior is observed for water in oil systems with particle-filled droplets with low viscosity ratios (Smith and van de Ven 1985b; Desse et al. 2011). In these systems, particles spend more time in the droplet tips during circulation. Consequently, particle clusters are ejected from the droplet tips at high shear rates.

In Fig. 9, a quantitative analysis is made by comparing the critical capillary number at which breakup occurs $\left(\mathrm{Ca}_{\text {crit }}\right)$. Additionally, the critical strain $\gamma_{\text {crit }}\left(=\dot{\gamma}_{\text {crit }} t_{\mathrm{b}}\right.$, with $t_{\mathrm{b}}$ the breakup time) and the dimensionless critical droplet length $L_{\mathrm{p}, \text { crit }} / 2 R$ are calculated (Fig. 10). However, results have to be interpreted with caution because the breakup mechanisms differ among the different particle systems, as already presented and discussed in Fig. 8. Therefore, data points in Figs. 9 and 10 of droplets that break up according to the tip streaming breakup mechanism are marked in the figure legend. The symbols refer to the symbols used in the first column of Fig. 8 .

$\mathrm{Ca}_{\text {crit }}$ values measured for the particle-free reference systems (RS) agree with the de Bruijn (1991) curve at viscosity ratios around 1 but deviate when reaching viscosity ratios close to 4 . It has to be noted however that Fig. 9 is a detailed figure because only a limited range of viscosity ratios is shown and both scales are linear. The inset of Fig. 9 therefore shows a complete breakup diagram of the critical capillary numbers determined in this work and the experimental data of Grace (1982) together with the de Bruijn curve (1991). This inset shows that the experimental data of Grace show similar

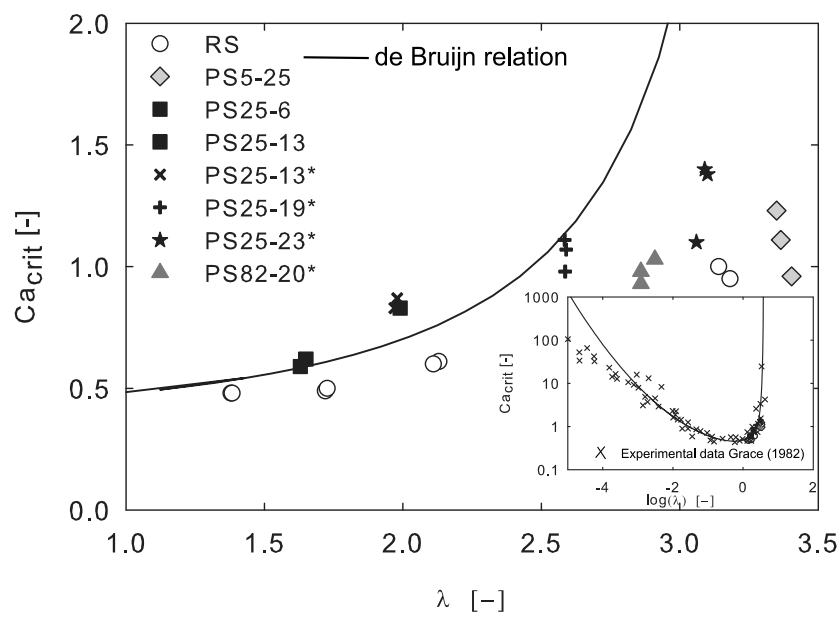

Fig. $9 \mathrm{Ca}_{\text {crit }}$ at which breakup occurs as function of bulk viscosity ratio $\lambda$. Data points with approximately the same viscosity ratio represent different droplets of the same particle or reference system. Entries indicated with $*$ refer to the tip streaming breakup mode. The same symbols are used in Figure 8 to indicate the specific breakup modes. Inset: Comparison with experimental data of Grace (1982)
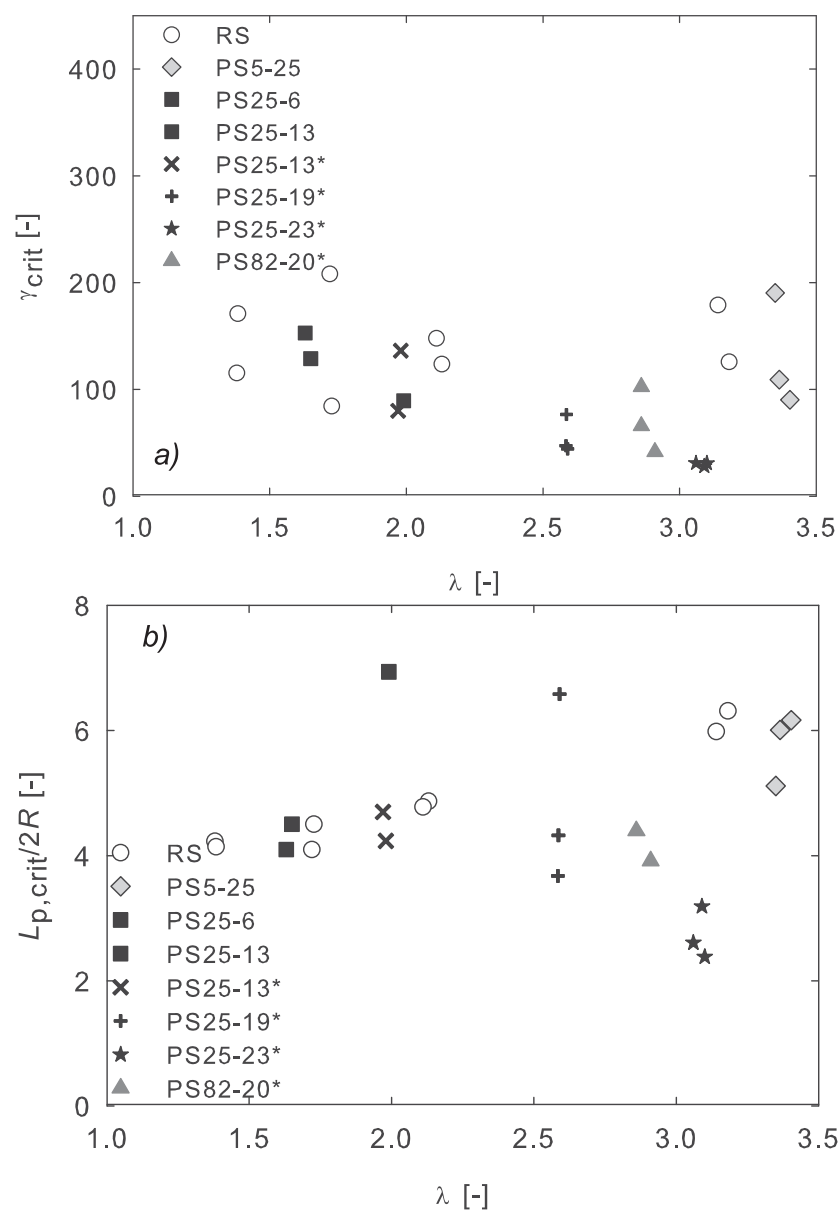

Fig. 10 (a) Critical strain $\gamma_{\text {crit }}$ and (b) critical droplet length $L_{\mathrm{p}, \text { crit }} / 2 R$ at which breakup occurs as function of bulk viscosity ratio $\lambda$. Data points with approximately the same viscosity ratio represent different droplets of the same particle or reference system. Entries indicated with * refer to the tip streaming breakup mode. The same symbols are used in Figure 8 to indicate the specific breakup mode

deviations from the de Bruijn fit in the investigated viscosity ratio range. Furthermore, the experimentally determined critical capillary numbers in the work of Zhao (2007) are also consistently lower than the predictions of de Bruijn (1991). The authors attributed these discrepancies to the fact that the gradual increase in capillary number used by Grace (1982) leads to higher critical Ca numbers than direct startup of flow, as applied here and in the work of Zhao (2007).

After the addition of high particle concentrations $(\geq$ 15 vol\%), the breakup behavior shows more variability. In general, the viscosity ratio is increased by the addition of particles compared with the original system RS-1.4 which results in this case in a higher value of $\mathrm{Ca}_{\text {crit }}$. Therefore, particle-filled droplets are more difficult to break and larger average droplet sizes are expected in blends. This particle effect is already extensively studied in literature for concentrated polymer blends (Zou et al. 2006; Gooneie et al. 2016) and diluted oil in water emulsions (Merkel et al. 2015). In addition, Kong et al. (2011) observed a higher critical breakup 
aspect ratio for single PA6 droplets containing silica nanoparticles which introduce viscoelastic effects in the dispersed phase. However, most of these works only compare the droplet size of particle-filled blends with that of the neat blend which has different viscous and/or elastic properties.

When we compare the $\mathrm{Ca}_{\text {crit }}$ values of PS25-6 and PS25-13 with the $\mathrm{Ca}_{\text {crit }}$ values of the corresponding reference systems with matched viscosity ratios, a slight increase is still observed. Hence, there is a small additional intrinsic effect of the particles that stabilizes the droplets further against breakup. Figure 10 shows that the critical strain and critical droplet length are in the same range as those of the respective reference systems.

At higher particle concentrations (PS25-19 and PS25-23), droplets break up according to the tip streaming mechanism, which results in a lower critical droplet length and lower critical strain. $\mathrm{Ca}_{\text {crit, }}$ on the other hand, is equal to or higher than $\mathrm{Ca}_{\text {crit }}$ observed for RS-3.2 (see Fig. 9). Hence, even though the central particle cluster does not break, the shift of the necking from the droplet center to the droplet tips caused by its presence, postpones breakup as compared with that in the neat RS-1.4 system.

Similar conclusions can be made for PS82-20, which also shows breakup by tip streaming. However, if smaller particles are added to the droplet phase (PS5-25), the breakup process is in most aspects comparable to the breakup behavior of the particle-free system RS-3.2 which approximately matches the viscosity ratio of PS5-25.

\section{Discussion}

In this section, the particle effects in the different experiments (steady-state deformation, transient droplet dynamics, and breakup behavior) are quantitatively compared with each other.

In general, we can state that particle systems behave in a similar way as a particle-free system with a higher viscosity ratio $\lambda^{*}$. For every data point of PS25-Y, PS5-25, and PS8220 presented in Fig. $4\left(\lambda^{*}\right.$ deformation $)$, Fig. $6 \mathrm{~b}\left(\lambda^{*}\right.$ startup $)$, and Fig. $7 \mathrm{~b}\left(\lambda^{*}\right.$ retraction $), \lambda^{*}$ is determined as the viscosity ratio which gives the same deformation $(L / 2 R)$ or characteristic time scale $\left(t_{\mathrm{i}}\right.$ or $\left.\tau\right)$ according to the MM model, shown as a full line in Figs. $4,6 \mathrm{~b}$, and $7 \mathrm{~b} . \lambda^{*}$ is not determined for the breakup experiments because a different breakup mode is observed for PS25-19, PS25-23, and PS82-20, which makes direct comparison difficult. For these systems, only tip streaming was observed, and it was impossible to break the particle cluster within the possible shear rate range. Therefore, we assume $\lambda^{*}$ for these systems larger than 3.8, which is the upper viscosity limit to break droplets in shear flow (Grace 1982; de Bruijn 1991).

To verify the hypothesis that particle-filled droplets can be treated as a homogeneous medium characterized by its bulk viscosity, $\lambda^{*}$ has to be compared with the bulk viscosity ratio $\lambda$ of the particle systems. Figure 11

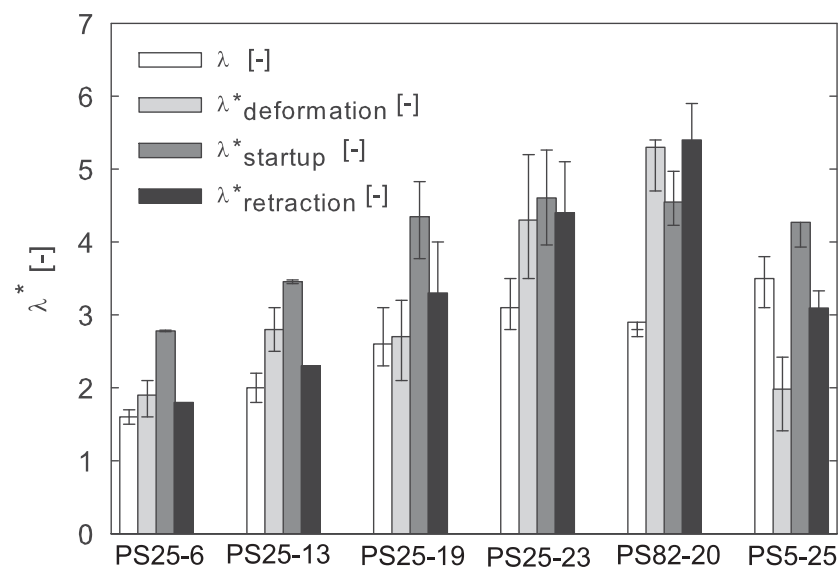

Fig. 11 Comparison of the bulk viscosity ratio $\lambda$ with a characteristic viscosity ratio $\lambda^{*}$ determined for all particle systems from experimental data of deformation, startup and retraction experiments. The error bars represent the minimal and maximal determined $\lambda^{*}$

summarizes the results. The error bars represent the minimal and maximal determined $\lambda^{*}$.

First, we focus on PS5-25, a system with a low particle over droplet size ratio $(r / R=0.02)$. As discussed in Fig. 4, the deformation of this system is slightly larger than the deformation of the corresponding reference system (RS-3.4) but still smaller than RS-1.4. Hence, $\lambda^{*}{ }_{\text {deformation }}$ is smaller than the bulk viscosity ratio $\lambda$ of PS5-25. Figure 11 also confirms that the retraction behavior of PS5-25 is comparable with the retraction behavior of a particle-free system with approximately the same viscosity ratio $\left({ }^{*}{ }_{\text {retraction }} \approx \lambda\right)$. As mentioned in the "Results" section, also the breakup behavior of PS5-25 agrees well with that of the corresponding reference system. These findings support the hypothesis that particles only influence the droplet dynamics by increasing the overall viscosity ratio of the system to the bulk viscosity ratio $\lambda$ determined by the particle concentration. Only for the startup experiments, the value of $\lambda^{*}$ is significantly higher than the bulk viscosity ratio $\lambda$ and more comparable with the other $20 \mathrm{vol} \%$ particle systems (PS25-23 and PS82-20).

Secondly, higher particle over droplet size ratios $(r / R \geq 0.1)$ are studied by PS25-Y and PS82-20. In contrast to PS5-25, these systems behave in a similar way as a particle-free system with a viscosity ratio slightly higher than their bulk viscosity ratio $\left(\lambda^{*}>\lambda\right)$. In addition, the particle clusters of PS25-19, PS25-23, and PS82-20 could not be broken, and hence, $\lambda *_{\text {breakup }}$ is larger than 3.8 , which is in all cases also larger than the bulk viscosity ratio $\lambda$. Furthermore, additional intrinsic particle effects like oscillations in deformation, asymmetrical droplet shapes, and tip streaming occur in these systems at high particle concentrations. These particle effects become more pronounced at higher particle concentrations or higher particle over droplet size ratios. Only the values of $\lambda^{*}$ determined from the startup experiments are independent of the particle size as PS5-25, PS25-23, and PS82-20 show similar 
values for $\lambda^{*}$ startup. Consequently, it seems that the startup dynamics are mainly governed by the particle concentration present in the droplet phase. It has to be noted however that the startup dynamics of the reference systems do not always agree with the MM model predictions. Therefore, we have to be careful with the interpretation of $\lambda^{*}$ startup.

Despite the fact that different experiments (steady-state deformation and transient dynamics) show similar trends, the exact values of $\lambda^{*}$ differ. Therefore, no general scaling law based on a corrected viscosity ratio can be composed to predict the complete droplet dynamics based on particle concentration and particle over droplet size ratio. Kaur and Leal (2010) made similar conclusions in their paper about the effect of particles in the matrix phase on droplet deformation and breakup. The authors stated that at low particle over droplet size ratios droplet deformation and breakup can be described by the bulk viscosity of the suspension, while at higher ratios the particles locally disturb the applied flow field and collide with the droplet. These last effects give rise to more fluctuations, asymmetric droplet shapes and scatter in the measured deformation and breakup behavior (Kaur and Leal 2010).

In our case, particles are located inside the droplet phase, and hence at higher particle over droplet size ratios or higher particle concentrations, also particle confinement inside the droplet will play a role. It is well-known that confinement leads to an increase of the viscosity of non-Brownian suspensions (Peyla and Verdier 2011). Moreover, particles will be excluded from a volume near the droplet interface as they do not cross the interface, which results in a higher particle concentration. Both types of confinement effects will be more pronounced for larger particles. As a result, these systems behave similar to a particle-free system with a viscosity ratio slightly higher than their bulk viscosity ratio $\lambda$. This effect is even observed at low particle concentrations, although very limited, and increases with particle concentration and particle size. Since the flow profile in the droplet is rather complex and may vary depending on whether steady-state conditions, startup, or retraction is considered, the effects of the particles on each of these processes can be slightly different, as demonstrated in our study.

\section{Conclusion}

In this work, the effects of non-Brownian particles localized in the droplet phase on single droplet dynamics are experimentally investigated in shear flow. The particle concentration and ratio of particle to droplet size are systematically varied. The results are compared with data of particle-free reference systems with comparable viscosity ratios.

In general, the particles increase the droplet phase viscosity and thus the viscosity ratio. The increase in viscosity ratio results in a suppressed droplet deformation, slower transient dynamics (after startup and cessation of shear flow), and a higher critical capillary number for breakup compared with the neat system.

At first glance, these effects can be predicted by the bulk viscosity ratio $\lambda$ of the system governed by the particle concentration. However, the presented results demonstrate that this is only possible at low particle over droplet size ratios $(r / R=0.02)$. The quantitative comparison of the dimensionless deformation axes $L / 2 R$ and characteristic time scales of the particle systems with those of the corresponding particlefree reference systems shows that the droplet dynamics of particle systems with $r / R \geq 0.1$ are not completely governed by their bulk viscosity ratio. Instead, the droplet dynamics are similar to those of a particle-free system with a viscosity ratio $\lambda^{*}$ slightly higher than their bulk viscosity ratio $\lambda$. Accordingly, the particle-filled droplets behave as more viscous than expected, although differences between $\lambda^{*}$ and $\lambda$ remain limited.

Furthermore, additional intrinsic particle effects come into play for particle over droplet size ratios exceeding 0.1 . For these particle systems, individual particles or a particle cluster bulging out of the droplet result in asymmetric droplet shapes and oscillations in the dimensionless droplet axes during steady-state shear flow. A different breakup mechanism which is similar to tip streaming for surfactant-covered droplets is also observed at high particle concentrations. Particle-free droplets are ejected from the droplet tips, while breakup of the central particle cluster seems to be impossible under the current operating window of the CR setup.

These intrinsic effects increase with particle concentration and particle over droplet size ratio. However, due to different intrinsic particle effects for steady-state deformation, startup, retraction, and breakup, it can be concluded that the complete droplet dynamics cannot be described by a single correction factor for the viscosity ratio.

Acknowledgments The authors would like to thank Jens Allard for his assistance with the confocal microscope.

Funding This research is funded by Bijzonder Onderzoeksfonds $\mathrm{KU}$ Leuven (GOA/15/007).

\section{Compliance with ethical standards}

Conflict of interest The authors declare that they have no conflict of interest.

Open Access This article is licensed under a Creative Commons Attribution 4.0 International License, which permits use, sharing, adaptation, distribution and reproduction in any medium or format, as long as you give appropriate credit to the original author(s) and the source, provide a link to the Creative Commons licence, and indicate if changes were made. The images or other third party material in this article are included in the article's Creative Commons licence, unless indicated otherwise in a credit line to the material. If material is not included in the 
article's Creative Commons licence and your intended use is not permitted by statutory regulation or exceeds the permitted use, you will need to obtain permission directly from the copyright holder. To view a copy of this licence, visit http://creativecommons.org/licenses/by/4.0/.

\section{References}

Amoabeng D, Roell D, Clouse KM, Young BA, Velankar SS (2017) A composition-morphology map for particle-filled blends of immiscible thermoplastic polymers. Polymer 119:212-223. https://doi.org/ 10.1016/j.polymer.2017.04.009

Bertrand T, Bonnoit C, Clément E, Lindner A (2012) Dynamics of drop formation in granular suspensions: the role of volume fraction. Granul Matter 14:169-174. https://doi.org/10.1007/s10035-0120318-3

Bharati A, Cardinaels R, Seo JW, Wübbenhorst M, Moldenaers P (2015) Enhancing the conductivity of carbon nanotube filled blends by tuning their phase separated morphology with a copolymer. Polymer 79:271-282. https://doi.org/10.1016/j.polymer.2015.09. 080

Bonnoit C, Bertrand T, Clément E, Lindner A (2012) Accelerated drop detachment in granular suspensions. Physics of Fluids 24:043304. https://doi.org/10.1063/1.4704801

Cardinaels R, Moldenaers P (2016) Morphology development in immiscible polymer blends. In: Guo Q (ed) Polymer Morphology: Principles, Characterization, and Processing p 348-373. https://doi. org/10.1002/9781118892756.ch19

de Bruijn RA (1991) Deformation and breakup of drops in simple shear flow. PhD Thesis, TU Eindhoven.

de Bruijn RA (1993) Tipstreaming of drops in simple shear flow. Chemical Engineering Science 48:277-284. https://doi.org/10. 1016/0009-2509(93)80015-I

Desse M, Wolf B, Mitchell J, Budtova T (2009) Experimental study of the break-up of starch suspension droplets in step-up shear flow. J Rheol 53:943-955. https://doi.org/10.1122/1.3123216

Desse M, Mitchell J, Wolf B, Budtova T (2011) Droplet deformation and break-up under shear: hydrocolloid solution vs. suspension of starch granules. Food Hydrocolloids 25:495-502. https://doi.org/10.1016/ j.foodhyd.2010.07.028

Elias L, Fenouillot F, Majesté JC, Cassagnau P (2007) Morphology and rheology of immiscible polymer blends filled with silica nanoparticles. Polymer 48:6029-6040. https://doi.org/10.1016/j.polymer. 2007.07.061

Elias L, Fenouillot F, Majesté JC, Alcouffe P, Cassagnau P (2008) Immiscible polymer blends stabilized with nano-silica particles: Rheology and effective interfacial tension. Polymer 49:43784385. https://doi.org/10.1016/j.polymer.2008.07.018

Filippone G, Dintcheva NT, La Mantia FP, Acierno D (2010) Using organoclay to promote morphology refinement and co-continuity in high-density polyethylene/polyamide 6 blends - effect of filler content and polymer matrix composition. Polymer 51:3956-3965. https://doi.org/10.1016/j.polymer.2010.06.044

Furbank RJ, Morris JF (2004) An experimental study of particle effects on drop formation. Phys Fluids 16:1777-1790. https://doi.org/10. $1063 / 1.1691034$

Gahleitner M, Kretzschmar B, Pospiech D, Ingolic E, Reichelt N, Bernreitner K (2006) Morphology and mechanical properties of polypropylene/polyamide 6 nanocomposites prepared by a twostep melt-compounding process. J Appl Polym Sci 100:283-291. https://doi.org/10.1002/app.23102

Geuskens G, Gielens JL, Geshef D, Deltour R (1987) The electrical conductivity of polymer blends filled with carbon-black. Eur
Polym J 27:1261-1264. https://doi.org/10.1016/0014-3057(91) 90063-T

Gooneie A, Nazockdast H, Shahsavan F (2015) Effect of selective localization of carbon nanotubes in PA6 dispersed phase of PP/PA6 blends on the morphology evolution with time, part 1: droplet deformation under simple shear flows. Polym Eng Sci 55:1504-1519. https://doi.org/10.1002/pen.24098

Gooneie A, Nazockdast H, Shahsavan F (2016) Effect of selective localization of carbon nanotubes in pa6 dispersed phase of PP/PA6 blends on the morphology evolution with time, part 2: relaxation of deformed droplets after cessation of flow. Polym Eng Sci 56:5160. https://doi.org/10.1002/pen.24191

Grace HP (1982) Dispersion phenomena in high viscosity immiscible fluid systems and application of static mixers as dispersion devices in such systems. Chem Eng Commun 14:225-277. https://doi.org/ $10.1080 / 00986448208911047$

Greco F (2002) Drop deformation for non-Newtonian fluids in slow flows. J Non-Newtonian Fluid Mech 107:111-131. https://doi.org/ 10.1016/S0377-0257(02)00144-1

Hong JS, Kim YK, Ahn KH, Lee SJ, Kim C (2006) Interfacial tension reduction in PBT/PE/clay nanocomposite. Rheol Acta 46:469-478. https://doi.org/10.1007/s00397-006-0123-1

Hong JS, Namkung H, Ahn KH, Lee SJ, Kim C (2006) The role of organically modified layered silicate in the breakup and coalescence of droplets in PBT/PE blends. Polymer 47:3967-3975. https://doi. org/10.1016/j.polymer.2006.03.077

Huang J (2002) Carbon black filled conducting polymers and polymer blends. Adv Polym Technol 21:299-313. https://doi.org/10.1002/ adv. 10025

Janssen PJA, Anderson PD (2007) Boundary-integral method for drop deformation between parallel plates. Phys Fluids 19:043602. https:// doi.org/10.1063/1.2715621

Kaur S, Leal LG (2010) Drop deformation and break-up in concentrated suspensions. J Rheol 54:981-1008. https://doi.org/10.1122/1. 3462306

Kelnar I, Khunová V, Kotek J, Kaprálková L (2007) Effect of clay treatment on structure and mechanical behavior of elastomer-containing polyamide 6 nanocomposite. Polymer 48:5332-5339. https://doi. org/10.1016/j.polymer.2007.06.062

Kennedy MR, Pozrikidis C, Skalak R (1994) Motion and deformation of liquid drops, and the rheology of dilute emulsions in simple shear flow. Computers \& Fluids 23:251-278. https://oi.org/10.1016/ 0045-7930(94)90040-X

Kobayashi H, Owen MJ (1995) Surface properties of fluorosilicones. Trends in Polymer Science 3:330-335. https://doi.org/10.1002/ masy.19940820114

Kong M, Huang Y, Chen G, Yang Q, Li G (2011) Retarded relaxation and breakup of deformed PA6 droplets filled with nanosilica in PS matrix during annealing. Polymer 52:5231-5236. https://doi.org/ 10.1016/j.polymer.2011.08.052

Kontopoulou M, Liu Y, Austin JR, Parent JS (2007) The dynamics of montmorillonite clay dispersion and morphology development in immiscible ethylene-propylene rubber/polypropylene blends. Polymer 48:4520-4528. https://doi.org/10.1016/j.polymer.2007. 05.068

Liu Y, Kontopoulou M (2006) The structure and physical properties of polypropylene and thermoplastic olefin nanocomposites containing nanosilica. Polymer 47:7731-7739. https://doi.org/10.1016/j. polymer.2006.09.014

Maffettone PL, Minale M (1998) Equation of change for ellipsoidal drops in viscous flow. Journal of non-Newtonian fluid mechanics 78:227241. https://doi.org/10.1016/S0377-0257(98)00065-2

Mehrabian S, Bussmann M, Acosta E (2015) Breakup of high solid volume fraction oil-particle cluster in simple shear flow. Colloids Surf A Physicochem Eng Asp 483:25-35. https://doi.org/10.1016/j. colsurfa.2015.06.054 
Mei Y, Li G, Moldenaers P, Cardinaels R (2016) Dynamics of particlecovered droplets in shear flow: unusual breakup and deformation hysteresis. Soft Matter 12:9407-9412. https://doi.org/10.1039/ C6SM02031C

Merkel T, Henne J, Hecht L, Gräf V, Walz E, Schuchmann HP (2015) Emulsification of particle loaded drops in simple shear flow. Colloids Surf A Physicochem Eng Asp 470:179-187. https://doi. org/10.1016/j.colsurfa.2015.01.080

Mewis J, Wagner NJ (2011) Colloidal suspension rheology. Cambridge University Press, New York

Minale M, Moldenaers P, Mewis J (1997) Effect of shear history on the morphology of immiscible polymer blends. Macromolecules 30: 5470-5475. https://doi.org/10.1021/ma9617330

Nuzzo A, Bilotti E, Peijs T, Acierno D, Filippone G (2014) Nanoparticleinduced co-continuity in immiscible polymer blends - a comparative study on bio-based PLA-PA11 blends filled with organoclay, sepiolite, and carbon nanotubes. Polymer 55:4908-4919. https://doi. org/10.1016/J.POLYMER.2014.07.036

Peyla P, Verdier C (2011) New confinement effects on the viscosity of suspensions. Europhys Letters 94:44001

Powell RL, Mason SG (1982) Dispersion by laminar flow. AlChE Journal 28:286-293. https://doi.org/10.1002/aic.690280218

Rallison JM (1984) The deformation of small viscous drops and bubbles in shear flow. Annual review of fluid mechanics 16:45-66. https:// doi.org/10.1146/annurev.fl.16.010184.000401

Ray SS, Pouliot S, Bousmine M, Utracki L (2004) Role of organically modified layered silicate as an active interfacial modifier in immiscible polystyrene/polypropylene blends. Polymer 45:8403-8413. https://doi.org/10.1016/j.polymer.2004.10.009

Smith PG, Van de Ven TGM (1985) Interactions between drops and particles in simple shear. Colloids and Surfaces 15:211-231. https://doi.org/10.1016/0166-6622(85)80072-X

Smith PG, van de Ven TGM (1985) Shear-induced deformation and rupture of suspended solid/liquid clusters. Colloids and Surfaces 15:191-210. https://doi.org/10.1016/0166-6622(85)80071-8

Taylor GI (1934) The formation of emulsions in definable fields of flow. Proceedings of the Royal Society A: Mathematical, Phys Eng Sci 146:501-523. https://doi.org/10.1098/rspa.1934.0169

Tjørve KMC, Tjrøve E (2017) The use of Gompertz models in growth analyses, and new Gompertz-model approach: an addition to the Unified-Richards family. PLoS ONE 12. https://doi.org/10.1371/ journal.pone. 0178691

Torza S, Cox RG, Mason SG (1972) Particle motions in sheared suspensions XXVII. Transient and steady deformation and burst of liquid drops. J Colloid Interface Sci 38:395-411. https://doi.org/10.1016/ 0021-9797(72)90255-X

Usta OB, Perchak D, Clarke A, Yeomans JM, Balazs AC (2009) Shear and extensional deformation of droplets containing polymers and nanoparticles. J Chem Phys 130:234905. https://doi.org/10.1063/1. 3153922
Vananroye A, Van Puyvelde P, Moldenaers P (2006) Effect of confinement on droplet breakup in sheared emulsions. Langmuir 22:39723974. https://doi.org/10.1021/la060442+

Vananroye A, Van Puyvelde P, Moldenaers P (2007) Effect of confinement on the steady-state behavior of single droplets during shear flow. J Rheol 51:139-153. https://doi.org/10.1122/1.2399089

Vananroye A, Cardinaels R, Van Puyvelde P, Moldenaers P (2008) Effect of confinement and viscosity ratio on the dynamics of single droplets during transient shear flow. J Rheol 52:1459-1475. https:// doi.org/10.1122/1.2978956

Vandebril S, Vermant J, Moldenaers P (2010) Efficiently suppressing coalescence in polymer blends using nanoparticles: role of interfacial rheology. Soft Matter 6:3353-3362. https://doi.org/10.1039/ B927299B

Velankar S, Moldenaers P (2002) Bidisperse polymeric mixtures for independent control of viscosity and elasticity. Rheol Acta 41:418 426. https://doi.org/10.1007/s003970100209

Verhulst K, Moldenaers P, Minale M (2007) Drop shape dynamics of a Newtonian drop in a non-Newtonian matrix during transient and steady shear flow. J Rheol 51:273. https://doi.org/10.1122/1. 2426973

Wahit MU, Hassan A, Rahmat AR, Lim JW, Mohd Ishak ZA (2006) Effect of organoclay and ethylene-octene copolymer inclusion on the morphology and mechanical properties of polyamide/ polypropylene blends. J Reinf Plast Compos 25:933-955. https:// doi.org/10.1177/0731684406063529

Yang H, Zhang X, Qu C, Li B, Zhang L, Zhang Q, Fu Q (2007) Largely improved toughness of PP/EPDM blends by adding nano-SiO2 particles. Polymer 48:860-869. https://doi.org/10.1016/j.polymer. 2006.12.022

Yoshida S, Trifkovic M (2019) Unraveling the effect of 3D particle localization on coarsening dynamics and rheological properties in cocontinuous polymer blend. Nanocomposites macromolecules 52:7678-7687. https://doi.org/10.1021/acs.macromol.9b01437

Zhang Q, Yang H, Fu Q (2004) Kinetics-controlled compatibilization of immiscible polypropylene/polystyrene blends using nano-SiO2 particles. Polymer 45:1913-1922. https://doi.org/10.1016/j.polyer. 2004.01.037

Zhao X (2007) Drop breakup in dilute Newtonian emulsions in simple shear flow: New drop breakup mechanisms. J Rheol 51:367-392. https://doi.org/10.1122/1.2714641

Zou H, Wang K, Zhang Q, Fu Q (2006) A change of phase morphology in poly(p-phenylene sulfide)/polyamide 66 blends induced by adding multi-walled carbon nanotubes. Polymer 47:7821-7826. https://doi.org/10.1016/j.polymer.2006.09.008

Publisher's note Springer Nature remains neutral with regard to jurisdictional claims in published maps and institutional affiliations. 